\title{
Patient's recognition level of medical terms as estimated by pharmacists
}

\author{
Yasuko Yoshida $\cdot$ Yoshitoku Yoshida
}

Received: 9 May 2014/ Accepted: 22 August 2014/Published online: 3 September 2014

(C) The Japanese Society for Hygiene 2014

\begin{abstract}
Objectives The role of pharmacists in the healthcare settings is expanding and pharmacists are expected to counsel patients and/or caregivers regarding the appropriate use of a drug. However, we believe that communication between healthcare providers and patients may be limited by overestimation of patients' recognition level of medical terms by providers. The purpose of this study was to clarify patients' recognition level of medical terms, mainly related to drugs, as estimated by pharmacists to contribute to improving risk communication in the medical care field.

Methods A total of 211 medical doctors and 212 pharmacists were surveyed. Differences between patients' recognition level of medical terms as estimated by medical doctors and pharmacists were assessed. In total, 90 medical terms were evaluated, including 57 medical terms from the National Institute for Japanese Language and an additional 33 medical terms.

Results Patient's recognition level of the selected medical terms as estimated by pharmacists was higher than that estimated by medical doctors.

Conclusions Compared with medical doctors, pharmacists tend to overestimate patients' recognition level of
\end{abstract}

Yasuko Yoshida and Yoshitoku Yoshida have contributed equally to this work.

\section{Y. Yoshida}

Graduate School of Pharmaceutical Sciences, Nagoya City

University, 3-1 Tanabe-dori, Mizuho-ku, Nagoya 467-8603,

Japan

e-mail: yy_miffy@yahoo.co.jp

Y. Yoshida $(\bowtie)$

Graduate School of Medicine, Nagoya University, 65

Tsurumaicho, Showa-ku, Nagoya 466-8550, Japan

e-mail: yoshidayoshitoku@med.nagoya-u.ac.jp medical terms. Therefore, pharmacists need to take greater care to ensure that their patients fully understand the risks and benefits of the drugs.

Keywords Risk communication - Perception gap · Shared decision-making $\cdot$ Medical term $\cdot$ Regulatory science

\section{Introduction}

In the medical care field, pharmaceutical products have a huge benefit that illness is treated through their bioactivity, but they also have risks, i.e., drug adverse effects. Concern over the drug adverse effects of new medicines is an important topic to address.

Furthermore, the concept of the shared decision-making has been evaluated for medical treatments [1], and recently the active participation of the patient in his or her treatment is also positively demanded in Japan [2].

Under such situations, we face perception gaps in pharmaceutical terms and related issues between patients and medical practitioners, which is one of the obstacles in practicing risk communication between them. To tackle this issue, Koch-Weser [3] examined medical word use in clinical encounters. The medical terms were checked to confirm their specific meaning in the healthcare field [4]. Chapman et al. [5] suggested that a substantial proportion of the laypeople do not understand phrases often used in cancer consultations. Chapple et al. [6] also suggested that the language used in the medical care field was often confusing and misunderstood by the families involved. Bass et al. [7] showed that the resident physicians overestimated the literacy abilities of their patients. There is a report on qualitative research from the viewpoint of risk 
evaluation in intensive care medicine [8]. In addition, the study says that the emphasis on appropriate medication counseling should not be limited to medications available only by prescription [9]. In Japan, we think there is still the specificity of "difference in recognition" and "difference in information" between patients and medical practitioners.

With regard to the information on adverse effects of medicine, a study was conducted in Japan by the National Institute for Japanese Language on the underlying recognition of medical terms by laypeople [10], but clinical trial terms and adverse effect terms were not examined at all in this research. Recently, clinical trials have been conducted vigorously and rigorously not only in Japan but also in China and other Asian countries [11, 12]. Therefore, in our previous study, we have conducted to elucidate the gaps in basic recognition of technical medical terms including clinical trial terms, adverse effect terms as well as the terms that the National Institute for Japanese Language examined between laypeople and medical doctors [13].

It has been introduced that the 6 years school period system of School of Pharmacy in Japan since 2006. In addition, according to Item 2 of Article 25 of the revision of the Pharmacist Law of 1996, pharmacists are required to counsel patients and/or caregivers regarding the appropriate use and potential side effects of the drugs [14]. Therefore, it is critical that pharmacists be able to accurately assess the health literacy of their patients to effectively communicate the necessary information. However, in our previous study, pharmacists were not surveyed as the subjects. Therefore, this time the patient's recognition level of medical terms estimated by pharmacists was surveyed.

In addition, Incorporated Administrative Agency Pharmaceuticals and Medical Devices Agency (PMDA) performs its three important services such as reviewing the application of new drugs, pharmaceutical products safety measures and the pharmaceutical products adverse effect damage relief based on the law of PMDA [15]. Given its increasing role in the pharmaceutical setting, it is extremely important that pharmacists recognize PMDA. Therefore, the recognition of this agency was also investigated.

\section{Methods}

The study protocol was approved by the Ethics Committee of the Graduate School of Medicine, Nagoya University prior to data collection.

\section{Subject}

Medical doctors and pharmacists were collected as the subject. The collection of the answer was performed under contract with NTT Rezonanto Co., Ltd. using an Internet survey monitored by Goo Research contractors. A two-step method was adopted for the selection of pharmacists, similar to the selection of medical doctors in our previous study [13]. First, a subgroup of healthcare workers was examined. Then, the respondents who answered that they were pharmacists were directed to subsequent questions.

The research was performed on the basis of the rules of NTT Rezonanto Co., Ltd. Samples representing $110 \%$ of the target number were collected and submitted to us after removing inappropriate samples. A total of 212 pharmacists were selected between Jan 18, 2012, and Jan 23, 2012, and a total of 211 medical doctors were selected between Feb 9, 2011, and Feb 11, 2011 [13].

Demographic data regarding pharmacists, such as their age, gender, location and scale of work place, experience of communicating directly with patients, and experience of participation in clinical trials, were collected in this study. Data regarding medical doctors, such as their age, gender, medical treatment department, scale of the medical institution, number of patients examined per day, and experience of participation in clinical trials, were collected in our previous study [13].

\section{Medical terms}

Differences between patients' recognition level of 90 medical terms as estimated by medical doctors and pharmacists were examined. The 90 medical terms consisted of 57 medical terms from the National Institute for Japanese Language [10] and an additional 33 medical terms [13].

The medical terms of the National Institute for Japanese Language were classified into 3 groups (A-C). Group A was "expressed in other words of vernacular speech" and included 13 medical terms such as ileus, evidence, and remission. Group B was "explained definitely" and was subdivided into 3 groups: Group B1 was "had to explain the correct meaning to the patients" and included 15 medical terms such as insulin, virus, and inflammation; Group B2 was "understood roughly but required more explanation to provide a reliable meaning" and included 17 medical terms such as malignant tumor, congestion, and depression; and Group B3 was "understood well, but the meanings that are used in the hospital are a little different from the meanings in vernacular speech. Therefore, avoiding confusion is important." and included the terms complications, shock, and anemia. Group $\mathrm{C}$ was categorized into 3 subgroups according to the study of the National Institute for Japanese Language [10]. Four medical care terms, i.e., informed consent, second opinion, guidelines, and clinical pass, were needed to explain important, new concepts. Three medical care terms, i.e., QOL, palliative care, and primary care, were needed to describe a new concept concerned with medical care and 
valuing everyday life. Two medical care terms for new medical instruments, MRI and PET were included to verify if laypeople knew whether they were receiving appropriate medical care. In total, 9 medical terms were listed. In this study, we combined all 3 subgroups of group $\mathrm{C}$ because they can all be categorized as terms needed to explain important, new concepts.

Furthermore, we targeted 7 medical terms that are primarily used in clinical trials, such as clinical investigation, GCP, and phase one clinical trial stage in group D. In addition, 26 medical terms related to adverse effects, such as anaphylaxis, Stevens-Johnson syndrome, and toxic necrolysis, were included in group E. In total, 90 medical terms were adopted as the target words. Perception of PMDA was also evaluated.

\section{Analysis}

Medical doctors and pharmacists evaluated each medical term using a scale of 1-5: (1) "I do not think that patients know," (3) "I cannot tell clearly whether patients know or not," and (5) "I think that patients know." This recognition was termed "patients' recognition level estimated by the medical doctors or pharmacists." In analyzing, (4) and (5) out of (1) to (5) were used as "I think that patients know".

Medical doctors and pharmacists also evaluated their recognition of PMDA using a scale of 1-3: (1) "I do not know PMDA," (2) "I have an experience to hear the PMDA," and (3) "I know PMDA." This recognition was termed "recognition level of the PMDA by the medical doctors or pharmacists." In analyzing, 3 was used as "I know the PMDA".

The Chi-square test was applied to analyze the differences between patient's recognition level of medical terms as estimated by medical doctors and by pharmacists. The Chi-square test was also applied to analyze the difference in the recognition level of PMDA by medical doctors and by pharmacists.

\section{Results}

Demographics

Table 1 shows the demographic characteristics of the survey respondents. In the present study, we obtained responses from 212 pharmacists (97 men and 115 women). For medical doctors, we used data from our previous study [13] in which responses were obtained from 211 medical doctors (194 men and 17 women). A statistically significant difference was observed in the age of medical doctors by gender. The majority of male medical doctors $(43.3 \%)$ were aged 40-49 years, whereas the majority of female doctors $(58.8 \%)$ were aged 30-39 years. On the other hand, though a statistically significant difference was also observed in the age of pharmacists by gender, the majority of male pharmacists $(34.0 \%)$ were $30-39$ and also the majority of female pharmacists $(35.7 \%)$ were aged 30-39 years. A significant difference was also observed in the clinical trial experience of pharmacists by gender; 35.1 and $21.7 \%$ male and female pharmacists, respectively, had clinical trial experience.

Difference between medical doctors and pharmacists with respect to patients' recognition level of the 90 medical terms

Table 2 shows patients' recognition level of the 90 medical terms as estimated by medical doctors and pharmacists.

Compared with medical doctors, pharmacists showed higher estimates of patients' recognition level for all 13 terms in group A, with $30.8 \%$ (4 out of 13) terms being estimated significantly higher. Furthermore, pharmacists showed higher estimates of patients' recognition level for all terms in group B, with the estimates being significantly higher for $40.0 \%$ (6 out of 15) terms in group B1, $52.9 \%$ (9 out of 17) terms in group B2, and $66.7 \%$ (2 out of 3 ) terms in group B3.

Among all 9 medical terms in group C, pharmacists' estimates of patients' recognition level of 8 medical terms excluding one term such as "MRI" were higher. The difference was statistically significant for only 1 of the 8 terms $(12.5 \%)$.

In group D, pharmacists showed higher estimates than medical doctors for all terms, and the difference was significant for $57.1 \%$ (4 out of 7) terms.

Lastly, in group E, pharmacists' estimates were higher for all terms except 2, "ventricular tachycardia" and "alveolar hemorrhage", and $50.0 \%$ (12 out of 24) terms were estimated significantly higher.

Difference between pharmacists with and without clinical trial experience with respect to patients' recognition level of 90 medical terms

Pharmacists with clinical trial experience ranked patients' recognition level at 70 out of 90 medical terms higher than pharmacists without experience. As shown in Table 3, the difference was statistically significant for 10 of these 70 terms. These 10 terms included 1 term from group C, 4 from group D, and 5 from group E. On the other hand, there was no statistically significant difference for the 20 medical terms that were estimated higher by pharmacists without clinical trial experience. 
Table 1 Demographic characteristics of respondents

\begin{tabular}{|c|c|c|c|c|c|c|c|}
\hline & \multicolumn{3}{|c|}{ Medical doctors } & & \multicolumn{3}{|l|}{ Pharmacists } \\
\hline & \multicolumn{3}{|l|}{ Sex } & & \multicolumn{3}{|l|}{ Sex } \\
\hline & $\begin{array}{l}\text { Male } \\
(n=194)\end{array}$ & $\begin{array}{l}\text { Female } \\
(n=17)\end{array}$ & & & $\begin{array}{l}\text { Male } \\
(n=97)\end{array}$ & $\begin{array}{l}\text { Female } \\
(n=115)\end{array}$ & \\
\hline Age & & & & Age & & & \\
\hline $20-29$ & $5(2.6 \%)$ & $2(11.8 \%)$ & $* *$ & $20-29$ & $13(13.4 \%)$ & $24(20.9 \%)$ & $* *$ \\
\hline $30-39$ & $39(20.1 \%)$ & $10(58.8 \%)$ & & $30-39$ & $33(34.0 \%)$ & $41(35.7 \%)$ & \\
\hline $40-49$ & $84(43.3 \%)$ & $4(23.5 \%)$ & & $40-49$ & $32(33.0 \%)$ & $26(22.6 \%)$ & \\
\hline $50-59$ & $56(28.9 \%)$ & $1(5.9 \%)$ & & $50-59$ & $16(16.5 \%)$ & $21(18.3 \%)$ & \\
\hline $60-69$ & $3(1.5 \%)$ & $0(0.0 \%)$ & & $60-69$ & $1(1.0 \%)$ & $3(2.6 \%)$ & \\
\hline 70 or more & $7(3.6 \%)$ & $0(0.0 \%)$ & & 70 or more & $2(2.1 \%)$ & $0(0.0 \%)$ & \\
\hline Institute & & & & Institute & & & \\
\hline Clinic (no beds) & $60(30.9 \%)$ & $4(23.5 \%)$ & n.s. & Own the pharmacy & $5(5.2 \%)$ & $0(0 \%)$ & n.s. \\
\hline Clinic (1-19 beds) & $12(6.2 \%)$ & $0(0.0 \%)$ & & Working at pharmacy & $52(53.6 \%)$ & $86(74.8 \%)$ & \\
\hline Hospital (20-99 beds) & $15(7.7 \%)$ & $0(0.0 \%)$ & & Clinic pharmacy & $1(1.0 \%)$ & $0(0.0 \%)$ & \\
\hline Hospital (100-199 beds) & $32(16.5 \%)$ & $3(17.6 \%)$ & & Hospital pharmacy (20-99 beds) & $3(3.1 \%)$ & $7(6.1 \%)$ & \\
\hline \multirow[t]{2}{*}{ Hospital (200 beds or more) } & $75(38.7 \%)$ & $10(58.8 \%)$ & & Hospital pharmacy (100 beds or more) & $33(34.0 \%)$ & $19(16.5 \%)$ & \\
\hline & & & & The others & $3(3.1 \%)$ & $3(2.6 \%)$ & \\
\hline Department & & & & Deliver the drug information & & & \\
\hline Internal medicine & $83(42.8 \%)$ & $9(52.9 \%)$ & n.s. & Yes & $95(97.9 \%)$ & $115(100 . \%)$ & n.s \\
\hline Surgery & $78(40.2 \%)$ & $6(35.3 \%)$ & & No & $2(2.1 \%)$ & $0(0.0 \%)$ & \\
\hline The others & $33(17.0 \%)$ & $2(11.8 \%)$ & & & & & \\
\hline No of outpatients/day & & & & Participation in clinical trials & & & \\
\hline 9 or less & $23(11.9 \%)$ & $3(17.6 \%)$ & n.s. & Yes & $34(35.1 \%)$ & $25(21.7 \%)$ & $*$ \\
\hline 10-19 Person & $33(17.0 \%)$ & $4(23.5 \%)$ & & No & $63(64.9 \%)$ & $90(78.3 \%)$ & \\
\hline 20-29 Person & $37(19.0 \%)$ & $3(17.6 \%)$ & & & & & \\
\hline \multirow[t]{2}{*}{ 30-39 Person } & $19(9.8 \%)$ & $4(23.5 \%)$ & & \multicolumn{4}{|c|}{ Type of participation in clinical trials ${ }^{\mathrm{a}}(n=59)$} \\
\hline & & & & Management of drugs & $32(94.1 \%)$ & $19(76.0 \%)$ & \\
\hline 40 Person or more & $82(42.3 \%)$ & n $(17.6 \%)$ & & Clinical & $5(14.7 \%)$ & $2(8.0 \%)$ & \\
\hline Participation in clinical trials & & & & Examinee & $2(5.9 \%)$ & $0(0.0 \%)$ & \\
\hline Yes & $116(59.8 \%)$ & $11(64.7 \%)$ & n.s. & & & & \\
\hline No & $78(40.2 \%)$ & $6(35.3 \%)$ & & The others & $3(8.8 \%)$ & $5(20.0 \%)$ & \\
\hline
\end{tabular}

${ }^{a}$ multiple answers were welcomed

n.s. not significant

$\chi^{2}$ test: ${ }^{* *} p<0.01, * p<0.05$

Difference between medical doctors and pharmacists with respect to recognition level of PMDA

There was a statistically significant difference between medical doctors and pharmacists with respect to recognition of PMDA: 27.0 and $65.1 \%\left(\chi^{2}\right.$ test: $\left.p<0.01\right)$, respectively. When evaluating in terms of demographic characteristics, there were statistically significant differences in recognition of PMDA between male $(74.2 \%)$ and female $(57.4 \%)$ pharmacists, between age groups of $<40$ years $\quad(73.0 \%)$ and $\geq 40$ years $(56.4 \%)$, and between pharmacists with clinical trial experience $(83.1 \%)$ and those without it $(58.2 \%)$. On the other hand, we could not find any significant difference among medical doctors in terms of demographic characteristics.

\section{Discussion}

Recently, Internet surveys have become widely used and accepted in the medical sociology field in Japan [16]. Internet surveys are useful because answers can be obtained in a rather short period of time by evaluating parameters previously registered with an Internet research company. 
Table 2 Difference between medical doctors and pharmacists with respect to patients' recognition level of the 90 medical terms
Group Medical term

Estimated by the medical doctors $(n=211)$

$\operatorname{Know}^{\mathrm{a}}(\%)$

Estimated by the pharmacists $(n=212)$

Know $^{\mathrm{a}}(\%)$

test

A

Critical condition

65.9

77.8

71.7

Prognosis

54.5

49.3

65.1

Aspiration

45.5

MRSA

44.1

Biopsy

37.9

Infiltration

33.2

Evidence

30.3

Remission

30.3

Deliria

30.3

Ileus

29.4

ADL

28.9

COPD

24.6

B1

Virus

78.2

Metabolic syndrome

74.9

Tumor

71.1

Insulin

70.6

Ulcer

68.7

Inflammation

66.4

Be taken as needed

62.1

Renal insufficiency

59.2

Geriatric health services

55.0 facilities

Steroid

52.1

Tumor marker

46.9

Group home

44.1

Symptomatic treatment

42.7

Sepsis

35.5

Connective tissue disease $\quad 33.2$

B2

Diabetes

85.3

82.5

Malignant tumor

81.5

Asthma

80.6

Arteriosclerosis

80.1

Depression

75.8

Heat stroke

72.0

Polyp

60.7

Brain death

59.7

Cirrhosis

58.8

Death with dignity

51.2

Chemotherapy

48.3

Jaundice

47.9

Anamnesis

42.7

Antibody

40.8

Clinical trial

Congestion

34.6

32.2

72.5

65.4

Complication

43.6
53.8

50.5

42.5

39.6

39.6

39.2

37.7

32.5

29.7

40.6

89.2

83.0

77.4

82.5

73.6

89.2

70.3

68.4

59.9

70.8

54.7

50.5

56.6

38.2

42.0

92.9

89.2

87.7

87.3

86.3

84.9

87.3

71.7

78.3

70.3

58.5

53.3

59.9

50.5

49.1

49.1

45.8

91.0

69.3

59.0

**

**

**

n.s.

n.s.

n.s.

n.s.

n.s.

n.s.

n.s.

n.s.

n.s.

**

**

*

n.s.

**

n.s.

**

n.s.

n.s.

n.s.

**

n.s.

n.s.

**

n.s.

n.s.

*

n.s.

n.s.

n.s.

n.s.

*

**

$*$

** 
Table 2 continued

\begin{tabular}{|c|c|c|c|c|}
\hline Group & Medical term & $\begin{array}{l}\text { Estimated by the medical doctors } \\
(n=211) \\
\operatorname{Know}^{\mathrm{a}}(\%)\end{array}$ & $\begin{array}{l}\text { Estimated by the pharmacists } \\
(n=212) \\
\operatorname{Know}^{\mathrm{a}}(\%)\end{array}$ & test \\
\hline \multirow[t]{9}{*}{$\mathrm{C}$} & MRI & 51.7 & 50.9 & n.s. \\
\hline & Informed consent & 44.5 & 46.7 & n.s. \\
\hline & Second opinion & 44.1 & 54.2 & $*$ \\
\hline & Palliative care & 35.1 & 40.1 & n.s. \\
\hline & Guidelines & 34.6 & 39.2 & n.s. \\
\hline & PET & 32.7 & 34.0 & n.s. \\
\hline & QOL & 28.9 & 36.3 & n.s. \\
\hline & Primary care & 25.1 & 26.9 & n.s. \\
\hline & Clinical pass & 20.4 & 21.7 & n.s. \\
\hline \multirow[t]{7}{*}{ D } & Clinical investigation & 29.9 & 39.2 & n.s. \\
\hline & Placebo & 25.6 & 36.8 & $*$ \\
\hline & Double blind trial & 22.7 & 33.0 & $*$ \\
\hline & Phase three clinical trial & 15.2 & 20.3 & n.s. \\
\hline & Phase one clinical trial & 14.7 & 23.1 & $*$ \\
\hline & Phase two clinical trial & 13.3 & 20.3 & n.s. \\
\hline & GCP & 8.5 & 18.4 & $* *$ \\
\hline \multirow[t]{26}{*}{$\mathrm{E}$} & $\begin{array}{l}\text { Anuresis/difficulty of } \\
\text { urination }\end{array}$ & 38.9 & 51.9 & $* *$ \\
\hline & Bleeding tendency & 37.9 & 53.8 & $* *$ \\
\hline & Hypothyroidism & 33.6 & 41.0 & n.s. \\
\hline & Thrombosis & 32.2 & 40.1 & n.s. \\
\hline & $\begin{array}{l}\text { Medicamentosus } \\
\text { stomatitis }\end{array}$ & 28.4 & 41.0 & $* *$ \\
\hline & Anaphylaxis & 26.1 & 38.7 & ** \\
\hline & Peripheral neuropathy & 24.2 & 32.5 & n.s. \\
\hline & Nephrotic syndrome & 24.2 & 31.6 & n.s. \\
\hline & Aplastic anemia & 21.8 & 34.9 & $* *$ \\
\hline & Ataxia & 20.9 & 28.8 & n.s. \\
\hline & Edema of lung & 20.4 & 24.5 & n.s. \\
\hline & Interstitial pneumonia & 19.9 & 31.1 & $*$ \\
\hline & Rhabdomyolysis & 19.9 & 31.1 & $*$ \\
\hline & Ventricular tachycardia & 19.9 & 19.8 & n.s. \\
\hline & $\begin{array}{l}\text { Stevens-Johnson } \\
\text { syndrome }\end{array}$ & 19.4 & 29.7 & $*$ \\
\hline & Agranulocytosis & 19.4 & 26.4 & n.s. \\
\hline & Guillain-Barre syndrome & 19.4 & 21.7 & n.s. \\
\hline & Angioedema & 19.0 & 25.5 & n.s. \\
\hline & Drug-related parkinsonism & 18.5 & 26.9 & $*$ \\
\hline & Malignant syndrome & 17.5 & 28.3 & * \\
\hline & Alveolar hemorrhage & 17.1 & 17.0 & n.s. \\
\hline & Pseudohyperaldosteronism & 16.1 & 25.5 & $*$ \\
\hline & Dyskinesia & 16.1 & 20.3 & n.s. \\
\hline & Toxic necrolysis & 15.6 & 23.1 & n.s. \\
\hline & Akathisia & 12.3 & 17.0 & n.s. \\
\hline & Hand-and-feet syndrome & 11.8 & 21.2 & $*$ \\
\hline
\end{tabular}

a 1 means "I do not think that patients know". 3 means "I cannot tell clearly whether the patient knows or not", 5 means "I think that patients know." In analyzing, 4 and 5 out of 1-5 were used as "I think that patients know"

n.s. not significant

$\chi^{2}$ test: $* * p<0.01, * p<0.05$
On the other hand, a face-to-face interview usually yields more detailed information such as the degree of the recognition level of medical terms. We believe that one of the limitations of this study is that responses were obtained using Internet surveys and may thus be superficial to some extent. 
Table 3 Difference between pharmacists with and without clinical trial experience with respect to patients' recognition level of the 90 medical terms

\begin{tabular}{lllll}
\hline Group & Medical term & \multicolumn{2}{l}{$\begin{array}{l}\text { Estimated by the } \\
\text { pharmacists }{ }^{\mathrm{a}}(\%)\end{array}$} \\
\cline { 3 - 5 } & & \multicolumn{2}{l}{ Experience of clinical trials } \\
\cline { 3 - 5 } & & $\begin{array}{l}\text { Yes } \\
(n=59)\end{array}$ & $\begin{array}{l}\text { No } \\
(n=153)\end{array}$ & Test \\
& & Know & Know & \\
\hline C & Clinical pass & 33.9 & 17.0 & $*$ \\
D & Phase one clinical trial & 35.6 & 18.3 & $*$ \\
D & Phase two clinical trial & 32.2 & 15.7 & $* *$ \\
D & Phase three clinical trial & 32.2 & 15.7 & $* *$ \\
D & GCP & 27.1 & 15.0 & $*$ \\
E & Ataxia & 39.0 & 24.8 & $*$ \\
E & Malignant syndrome & 39.0 & 24.2 & $*$ \\
E & Drug-related parkinsonism & 37.3 & 22.9 & $*$ \\
E & Ventricular tachycardia & 32.2 & 15.0 & $* *$ \\
E & Alveolar hemorrhage & 27.1 & 13.1 & $*$ \\
\hline
\end{tabular}

a 1 means "I do not think that patients know". 3 means "I cannot tell clearly whether the patient knows or not", 5 means "I think that patients know." In analyzing, 4 and 5 out of 1-5 were used as "I think that patients know"

n.s. not significant

$\chi^{2}$ test: $* * p<0.01, * p<0.05$

Our previous study showed that the eldest citizen group had the highest understanding of the 90 medical terms selected [13], indicating that the elderly persons who are computer literate enough to participate in the Internet survey are also the most healthcare literate. It is possible that this trend also applies to medical doctors and pharmacists. Therefore, this can be considered another limitation of this study.

Our previous study indicated that there was a huge gap in laypeople's recognition level of medical terms and the recognition level as estimated by medical doctors, particularly with respect to more difficult medical terms [13].

This study showed that pharmacists tend to estimate patients' recognition level of medical terms higher than medical doctors. More than $50 \%$ of medical terms which has been observed the statistically significant differences between medical doctors and pharmacists were group B2, B3 and D. Group B2 was defined as "understood roughly but required more explanation to provide a reliable meaning" and Group B3 was defined as "understood well, but the meanings that are used in the hospital are a little different from the meanings in vernacular speech". We think recently pharmacists are requested to explain the information of drug to a patient rigorously and vigorously but even now they do not have an enough chance to check whether patient's recognition is appropriate or not. Therefore, pharmacists need to consider that even when they explain the easier medical to patients, they need to explain them with the viewpoint of avoiding confusion. Given that one of the most important duties of a pharmacist is to provide information and counseling on the drug when it is dispensed [14], pharmacists need to be aware that patients' recognition level is likely to be much lower than what they perceive.

Group D was defined as "medical terms that are primarily used in clinical trials". Our previous study showed that this group was the most difficult group for patients. In addition, in this study, pharmacists with clinical trial experience tended to estimate patients' recognition level of medical terms higher than pharmacists without clinical trial experience. In addition, the 10 medical terms with statistically significant difference consisted of 1 medical term in group $\mathrm{C}$ of new concepts, 4 medical terms in group D of the clinical trial-related terms, and 5 medical terms in group $\mathrm{E}$ of the medical care terms related to adverse effects. We think that pharmacists deliver the drug information to patients who have already seen their medical doctors and have been familiar with the medical words. This fact influences the patients' recognition level which pharmacists estimated, especially for the pharmacists with clinical trial experience because they have a chance to talk about the patients who were taken of informed consent by medical doctors to be involved in the clinical trials. The main role of pharmacists in clinical trials is to manage the drugs that are used to evaluate the efficacy and safety. Thus, it is possible that these pharmacists had so many opportunities to hear or see the clinical trial-related terms and adverse effect terms during the clinical trials that they estimated patients' recognition level to be higher.

Conversely, our previous study showed that patients' recognition level as estimated by medical doctors with clinical trial experience was lower than that by medical doctors without clinical trial experience [13]. Based on these results, we believe that clinical trials foster better communication between medical doctors and patients.

Furthermore, pharmacists need to convey not only the information on the safety and efficacy of drugs but also, if necessary, information regarding the role of PMDA to a patient so that they can be prepared to deal with potential adverse effects.

With the development of more targeted pharmaceutical therapeutics, the role of pharmacists in the healthcare settings is expanding. Pharmacists are now expected to act as clinical research coordinators in medical institutes such as Incorporated Administrative Agency National Hospital Organization of Japan [17]. Moreover, pharmacists work 
more closely than ever with other healthcare providers, including medical doctors and nurses, in hospital environments in Japan [18].

With the increasing recognition of the value of shared decision-making in the medical field [1] and the consequent increasing solicitation of active patient involvement in his/her treatment in Japan [2], pharmacists as well as medical doctors need to be aware of the limitations of patients' recognition level of medical terms. Patients and/ or caregivers are unlikely to fully comprehend pharmaceutical terms and the related issues. Therefore, pharmacists as well as medical doctors must take great care to do the risk communications of pharmaceutical therapies vigorously and rigorously.

This is particularly true for pharmacists who tend to overestimate the understanding of their patients. Therefore, we believe that training on effective communication with patients should be added to the educational curriculum of pharmacists that has been reformed as the 6-year education period system in Japan since 2006 with viewpoint of regulatory sciences.

Acknowledgments This study was partially supported by JSPS KAKENHI Grant Number 22590584 from the Japan Society for the Promotion of Science.

Conflict of interest The authors declare that they have no conflicts of interests.

\section{References}

1. Politi MC, Clark MA, Ombao H, Dizon D, Elwyn G. Communicating uncertainty can lead to less decision satisfaction: a necessary cost of involving patients in shared decision making? Health Expect. 2011;13:84-91.

2. Kitano M. What's the difference? Comparison of American and Japanese medical practice. Keio J Med. 2007;56:96-101.

3. Koch-Weser S, DeJong W, Rudd RE. Medical word use in clinical encounters. Health Expect. 2009;12:371-82.

4. Bell C. A hundred years of Lancet language. Lancet. 1994; 2:1453.
5. Chapman K, Abraham C, Jenkins V, Fallowfield L. Lay understanding of terms used in cancer consultations. Psychooncology. 2003;12:557-66.

6. Chapple A, Campion P, May C. Clinical terminology: anxiety and confusion amongst families undergoing genetic counseling. Patient Educ Couns. 1997;32:81-91.

7. Bass PF, Wilson JF, Griffith CH, Barnett DR. Residents' ability to identify patients with poor literacy skills. Acad Med. 2002;77:1039-41.

8. Eidesen K, Sollid SJM, Aven T. Risk assessment in critical care medicine: a tool to assess patient safety. J Risk Res. 2002; 12:281-94.

9. Ledford CJW, Childress MA, Ledford CC, Mundy HD. The practice of prescribing: discovering differences in what we tell patients about prescription medications. Patient Educ Couns. 2014;94:255-60.

10. The National Institute for Japanese Language. Proposal to facilitate understanding of the "word of hospital" 2009. http://www. ninjal.ac.jp/byoin/teian/ruikeibetu/teiango/index.html (in Japanese). Accessed 28 Aug 2014.

11. Yoshida Y, Zhang Y, Yoshida Y, Ma D, Wang P. Current situation of clinical trials in Beijing, China. Contemp Clin Trials. 2012;33(4):583-8.

12. Yoshida Y, Xue D, Yoshida Y, Zhang Y, Ma D, Sato Y, Wang P. Current Situation of Clinical Trials in Beijing and Shanghai, China. Int J Clin Pharmacol Ther. 2013;51(5):433-40.

13. Yoshida Y, Yoshida Y, Motoyoshi T, Saito M, Saito MA, Hayase T. Study of perception gaps in pharmaceutical terms and related issues between laypeople and medical practitioners. Nihon Eiseigaku Zasshi. 2013;68:126-37 (in Japanese).

14. Revision of the pharmacist law (dated August 10, 1960, No. 146) dated June 26, 1996, No. 104.

15. Establishment of the law of Incorporated Administrative Agency Pharmaceuticals and Medical Devices Agency dated December 20, 2002, No. 192.

16. Adachi $\mathrm{M}$. Internet investigation about treatment and the disease recognition in the asthmatic. Allergol Immuno. 2011;18:1034-45 (in Japanese).

17. Misaki T, Nakazawa M, Tsutsumi K, Kayanuma T, Kamiyama Y, Fukasawa K, et al. Participation of pharmacist CRC in practical on-site training-a survey by questionnaires for pharmacy students. Yakugaku Zasshi. 2009;129:1265-74 (in Japanese).

18. Matsubara K, Toyama A, Satoh H, Suzuki H, Awaya T, Tasaki Y, et al. Longer working hours of pharmacists in the ward resulted in lower medication-related errors-survey of national university hospitals in Japan. Yakugaku Zasshi. 2011;131:635-41 (in Japanese). 